\title{
Size effect investigation of indentation response of stiff film/compliant substrate composite structure
}

\author{
Yanwei Liu ${ }^{\mathrm{a}}$, Hansong Ma ${ }^{\mathrm{b}}$, Yueguang Wei ${ }^{\mathrm{a}, *}, \mathrm{Pu}$ Chen ${ }^{\mathrm{a}}$ \\ a Department of Mechanics and Engineering Science, College of Engineering, Peking University, Beijing, 100871, China \\ ${ }^{\mathrm{b}}$ State-Key Laboratory of Nonlinear Mechanics, Institute of Mechanics, Chinese Academy of Sciences, Beijing, 100190, China
}

\section{A R T I C L E I N F O}

\section{Article history:}

Received 7 August 2019

Revised 2 February 2020

Accepted 9 February 2020

Available online 13 February 2020

\section{Keywords:}

Stiff film/compliant substrate composite structure

Indentation response

Gradient effect

Integral transformation

Material length scale

\begin{abstract}
A B S T R A C T
The stiff film/compliant substrate composite structure with a high modulus ratio finds a wide range of applications in production and in scientific research, and its indentation behavior cannot be described by the traditional theory when the film thickness is reduced from the millimeter scale to the micrometer or nanometer scale. In order to better understand the trans-scale indentation response of the composite structure caused by the reduction in the film thickness, this problem is solved analytically with the strain gradient theory and integral transformation. The gradient effect on the indentation response of the system is assessed in detailed from three aspects: load-displacement relationship, surface topography and distribution of bending moment on the film. In addition, the influences of film thickness, modulus ratio of the film to the substrate, contact radius and Poisson's ratio on the gradient effect are investigated. It is found that the gradient effect on the indentation response of the system, which is not sensitive to the contact radius and Poisson's ratio, is related not only to the film thickness but also to the modulus ratio of the film to the substrate. Based on the above analysis, a dimensionless number $(\mathrm{g} / \mathrm{l})$ is proposed to evaluate the gradient effect on the indentation behaviors of the system. And with the help of the dimensionless number, a new simple and accurate method for measuring the material length scale is proposed. Our research provides a theoretical basis for an in-depth understanding of the gradient effects on the indentation response of the stiff film/compliant substrate system and for the measurement of the material length scale.
\end{abstract}

() 2020 Elsevier Ltd. All rights reserved.

\section{Introduction}

Due to their wide applications in flexible electronic devices (Hou et al., 2014; Wang et al., 2012; Yin et al., 2010), protective coatings (Thobor-Keck et al., 2005; Zheludkevich et al., 2005), biomimetic composites (Chen and Lu, 2012; Fang et al., 2010), and so on, the stiff film/compliant substrate structures have received extensive attention. As the core structure in these applications, the physical properties of the stiff film/compliant substrate composite structures, especially their mechanical properties, have a significant impact on the functionality and stability of those devices. For example, the electronic skin fabricated by covering graphene on a polymer substrate undergoes different deformations when subjected to different external loads, which in turn induces different electrical signals (Hou et al., 2014); when the protective coating is destroyed by external loads, its corrosion resistance and wear resistance are lost (Fu et al., 2016). In addition, this com-

\footnotetext{
* Corresponding author.

E-mail addresses: 1601214764@pku.edu.cn (Y. Liu), weiyg@pku.edu.cn (Y. Wei).
}

posite structure is also widely used in laboratory measurements. For example, Cao et al. measured the mechanical properties of the single-layer graphene by means of this structure (Cao et al., 2018; Niu et al., 2018); through the theoretical analysis of the indentation response of this structure, Liu et al. proposed an accurate method for obtaining the mechanical properties of nanofilms when considering the viscoelasticity of the substrate (Liu et al., $2019 b)$. In those application, the modulus ratio of the film to the substrate is greater than $10^{2}$. Therefore, an in-depth understanding of the mechanical behaviors of the stiff film/compliant substrate system with a high modulus ratio contributes to its better application.

For the stiff film/compliant substrate composite structure, one of the main load conditions is the out-of-plane pressure which is similar to the indentation force. Consequently, a variety of studies on its indentation behaviors have been carried out (Bucaille et al., 2002; Burnett and Rickerby, 1987; Cao et al., 2018; Doerner and Nix, 2011; Gao et al., 1992, 2008; Jönsson and Hogmark, 1984; Lesage et al., 2005; Liu et al., 2019a; Ramsey et al., 1991; Tuck et al., 2001). For example, to accurately describe the inden- 
tation behaviors of film/substrate composite structures, empirical solutions (Doerner and Nix, 2011; King, 1987), approximate solutions (Gao et al., 1992; Xu and Pharr, 2006), and analytical solutions (Gao et al., 2008; Liu et al., 2019a) were established. For example, according to various experimental results, Doerner and Nix (Doerner and Nix, 2011) first proposed an empirical model, which was further improved by King (King, 1987) to improve its range of applicability. Based on the perturbation analysis, Gao et al. provided an approximate model (Gao et al., 1992), which was modified by $\mathrm{Xu}$ and Pharr to improve its accuracy (Xu and Pharr, 2006). Gao et al. derived an analytical relationship between surface tractions and displacements by Green's function in Fourier space. To describe the hardness of the film/substrate composite structure more accurately, the predictive models (Burnett and Rickerby, 1987; Jönsson and Hogmark, 1984; Lesage et al., 2005) and descriptive model (Tuck et al., 2001) have been developed. In addition, the effects of the pile-up, elastic and plastic inhomogeneity, and the modulus ratio on the indentation behaviors of the film/substrate composite structure were investigated by simulations and experiments (Cao et al., 2018; Saha and Nix, 2002; Vanimisetti and Narasimhan, 2006).

In recent years, with the development of microelectromechanical systems or nanoelectromechanical systems, the film/substrate composite structure, as a key component of the system, is becoming smaller and smaller, and the thickness of the film mounted on the compliant substrate is reduced from the millimeter scale to the micrometer or nanometer scale. For example, the thickness of the film mounted on different substrates to fabricate the nano transistor (Wang et al., 2012), flexible field emission device (Yin et al., 2010), and electronic skin (Ho et al., 2016) is usually in the nanoscale. Therefore, more and more attention has been paid to the mechanical behaviors of ultra-thin film/substrate systems. However, the mechanical behavior of materials with a small scale, especially in the microscale or nanoscale, cannot be described adequately by the traditional theory. In order to better predict the indentation size effect of mechanical behaviors of materials and film/substrate system, the strain gradient theories and discrete dislocation plasticity analysis have been applied. For example, Begley and Hutchinson used the Fleck-Hutchinson strain gradient plasticity theory which is based on the thermodynamics frame (Fleck and Hutchinson, 1997) to describe the decreasing hardness with increasing depth of indentation at shallow indentations for metals by adopting the finite element calculation (Begley and Hutchinson, 1998); Saha et al. used the mechanism-based strain gradient (MSG) plasticity theory which is based on the Taylor dislocation model (Gao et al., 1999) to explain the decreasing hardness with increasing depth of indentation and the rise in hardness that occurs when the indenter tip approaches the soft film/hard substrate interface (Saha et al., 2001). With the help of MSG theory, Zhang et al. studied the indentation response of the stiff film/compliant substrate system, and the results show that the strain gradient effect in the compliant substrate is insignificant, but that in the stiff film is important in shallow indentation, and the strain gradient effect in the stiff film disappears rapidly as the indentation depth increases (Zhang et al., 2007); based on the discrete dislocation plasticity analysis, Balint et al. studied the size effect of the indentation of single crystal films on a rigid substrate by a rigid wedge indenter, and found that for the thick film $(t=10 \mu \mathrm{m}$ and $t=50 \mu \mathrm{m})$, the hardness decreases with the increasing indentation depth, but for the thin film $(t=2 \mu \mathrm{m})$, the hardness first decreases with the increasing indentation depth and subsequently increases with the increasing indentation depth as the plastic zone reaches the rigid substrate (Balint et al., 2006); with the discrete dislocation plasticity analysis, $\mathrm{Xu}$ et al. investigated the size effect of thin single crystal films bonded to a rigid substrate indented by a rigid wedge for different values of film thickness and dislocation source den- sity, and a modified form of the scaling law for the dependence of hardness on indentation depth is proposed (Xu et al., 2019). In addition, Chen et al. analyzed the effects of the substrate and film size on the indentation behaviors of the film/substrate system by means of experiments and simulations (Chen et al., 2007). In the above studies, the modulus ratio of the film to the substrate is small $\left(E_{f} / E_{s}<10\right)$ or the film is bonded on the rigid substrate, so the film will have obvious plastic deformation when the indenter is pressed into the film/substrate system. Therefore, the strain gradient plasticity theory or the discrete dislocation plasticity theory is used to investigate the size effect of the film/substrate system. However, for the stiff film/compliant substrate system with a high modulus ratio $\left(E_{\mathrm{f}} / E_{\mathrm{s}}>10^{2}\right)$, the elastic deformation in the film is dominant when the indentation depth is smaller than the film thickness (Cao et al., 2018; Liu et al., 2019a, 2019b). At this time, the strain gradient plasticity theory and the discrete dislocation plasticity theory are not applicable for this case, and a better theory which can describe the indentation size effect of the stiff film/compliant substrate system correctly needs to be used, such as the strain gradient elasticity theory. In addition, until now, the indentation size effect of the stiff film/compliant substrate system with a high modulus ratio $\left(E_{\mathrm{f}} / E_{\mathrm{s}}>10^{2}\right)$ is rarely reported. And it is not clear that how the gradient effect influences the indentation response of the stiff film/compliant substrate composite structure with a high modulus ratio and which factors are related to the gradient effect. What's more, if the gradient effect exists in the indentation response of the stiff film/compliant substrate system, how can we effectively use it?

In this paper, in order to have a deep understanding of the indentation size effect of the stiff film/compliant substrate system, a detailed study of this system from a theoretical point of view has been conducted based on the simplified strain gradient elastic theory due to Mindlin with just three constants. The arrangement of this paper is as follows: In Section 2, a closed-form indentation solution of the stiff film/compliant substrate composite structure is derived with the strain gradient elastic theory and Hankel transformation. On the basis of this solution, the gradient effect on the indentation response is investigated from three aspects: the loaddisplacement relationship, the surface morphology, and the bending moment distribution on the film in Section 3. In Section 4, the influences of film thickness, modulus ratio of the film to the substrate, contact radius and Poisson's ratio on the indentation gradient effect are analyzed, systematically. Based on the above analysis, a dimensionless number $(\mathrm{g} / \mathrm{l})$ is proposed to describe the gradient effect. At the same time, with the help of the dimensionless number, a new method to measure the material length scale is established. At last, the main results and conclusions are summarized in Section 5. Our research allows the reader to gain an in-depth understanding of the indentation gradient effects of stiff film/compliant substrate composite structures. At the same time, it provides a new convenient method for measuring the material length scale.

\section{Theory and model}

For the indentation response of film/substrate system, the gradient effect in the film is important in shallow indentation depth, but that in the substrate is insignificant (Zhang et al., 2007), namely, the size effect of the indentation of this system is mainly due to the gradient effect of the film. Therefore, it is reasonable to assume the stiff film as a strain gradient material and the substrate as a traditional material. In addition, the bending stiffness of the film has a huge influence on the indentation behavior of the film/substrate system, especially when the modulus ratio of the film to the substrate is relatively high. (Liu et al., 2019a; Vanimisetti and Narasimhan, 2006). And there will be obvious sur- 


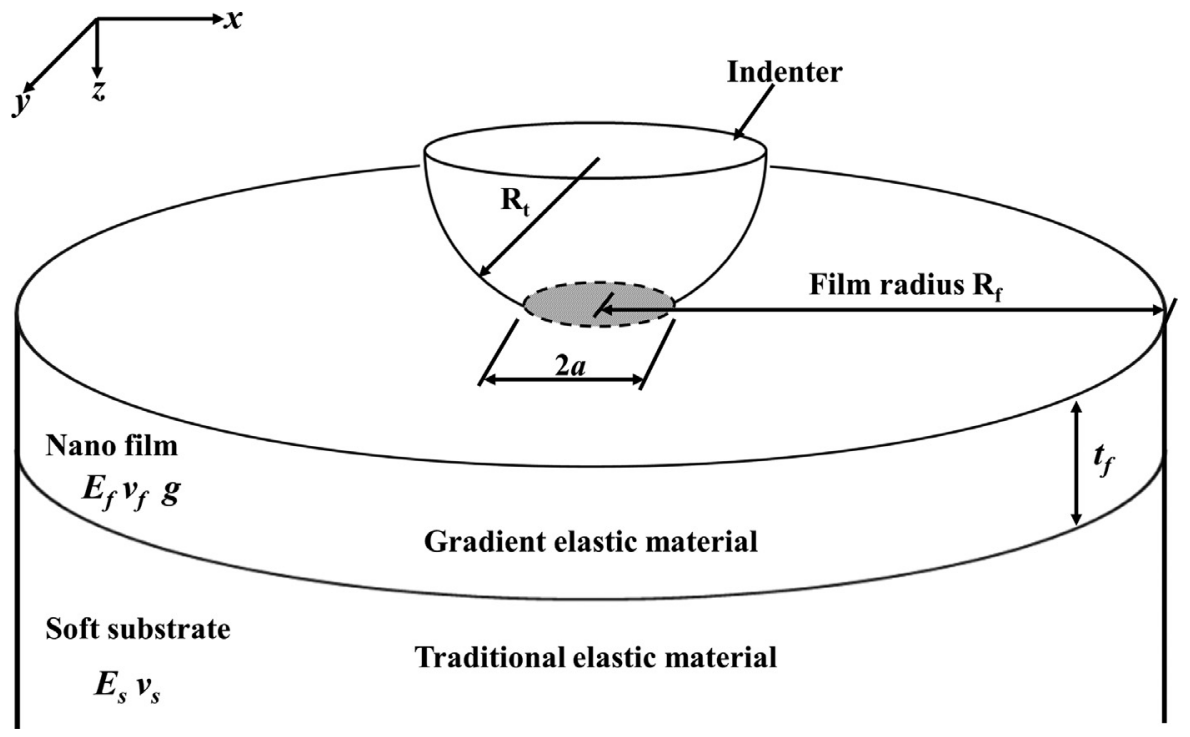

Fig. 1. The diagram of the indentation model of the gradient elastic film resting on a soft elastic substrate.

face sedimentation due to the high modulus ratio of the film to the substrate. At this time, the deformation of the film can be regarded as the bending of the plate mounted on a foundation. In addition, when the indentation depth is small, namely, the deflection of the film is small, the mechanical behaviors of the film can be considered to be elastic. Besides, because of the presence of the stiff film, there is no stress concentration in the compliant substrate (Cao et al., 2018). Therefore, the substrate can also be considered to be elastic. Based on the above analysis, we can know that the elastic deformation in the film and the substrate is dominant and the strain gradient plasticity theory and the discrete dislocation plasticity theory are not applicable for the indentation size effect of the stiff film/compliant with a high modulus ratio. In order to describe the indentation size effect of the system more correctly, the strain gradient elasticity theory is used to establish the model, and the indentation problem of the stiff film/compliant substrate system can be regarded as the bending of an infinite gradient elastic plate mounted on a semi-infinite elastic foundation.

The diagram of the gradient elastic stiff film/soft elastic substrate system is shown in Fig. 1. In this section, the closed-form indentation solution of the gradient elastic stiff film/soft elastic substrate composite structure is obtained by combining the governing equation of the gradient elastic plate with the solution of the semi-infinite elastic foundation under an axisymmetric load with the help of Hankel transformation.

\subsection{The governing equation for the gradient elastic plate mounted on a compliant substrate}

First, based on the variational principle, the governing equations for the gradient elastic plate are obtained. The simplified theory of strain gradient linear elasticity due to Mindlin (Mindlin, 1964) with five constants is that with just three constants containing two Lame constants and one characteristic length of the solid (Altan and Aifantis, 1997). The constitutive equations for this simple gradient elastic theory are

$$
\begin{aligned}
\sigma & =2 \mu \varepsilon+\lambda \operatorname{tr} \varepsilon I \\
\tau & =\mathrm{g}^{2} \nabla \sigma \\
\sum & =\sigma-\nabla \cdot \tau
\end{aligned}
$$

In the above, $\sigma, \tau$, and $\Sigma$ are the classical Cauchy stress, the couple stress, and the total stress tensors, respectively (A detailed explanation of the couple stress can be seen in the appendix.). $\varepsilon=$ $1 / 2(\nabla u+u \nabla)$ and $\operatorname{tr} \varepsilon=\nabla \cdot u$ are the strain tensor and its trace. $I$ is a unit tensor. $g$ is the characteristic length of the solid, and its value is at about the micron meter, by which one can describe the size effect due to strain gradient effect. $\lambda$ and $\mu$ are the classical Lame constants.

According to Kirchhoff's hypothesis, the stress in the plane $\left(\sigma_{x x}, \sigma_{y y}, \sigma_{x y}, \tau_{x x x}, \tau_{x x y}, \tau_{y y x}, \tau_{y y y}, \tau_{x y x}, \tau_{x y y}\right)$ is only considered. Based on Eq. (1), the plane stress can be written in terms of Cartesian plane coordinates $\mathrm{x}$ and $\mathrm{y}$ as

$$
\begin{aligned}
\sigma_{x x} & =\frac{E_{f}}{1-v_{f}^{2}}\left(\varepsilon_{x x}+v \varepsilon_{y y}\right), \tau_{x x x}=g^{2} \frac{E_{f}}{1-v_{f}^{2}} \frac{\partial\left(\varepsilon_{x x}+v \varepsilon_{y y}\right)}{\partial x}, \\
\tau_{x x y} & =g^{2} \frac{E_{f}}{1-v_{f}^{2}} \frac{\partial\left(\varepsilon_{x x}+v \varepsilon_{y y}\right)}{\partial y} \\
\sigma_{y y} & =\frac{E_{f}}{1-v_{f}^{2}}\left(\varepsilon_{y y}+v \varepsilon_{x x}\right), \tau_{y y x}=g^{2} \frac{E_{f}}{1-v_{f}^{2}} \frac{\partial\left(\varepsilon_{y y}+v \varepsilon_{x x}\right)}{\partial x}, \\
\tau_{y y y} & =g^{2} \frac{E_{f}}{1-v_{f}^{2}} \frac{\partial\left(\varepsilon_{y y}+v \varepsilon_{x x}\right)}{\partial y} \\
\sigma_{x y}= & \frac{E_{f}}{1+v_{f}} \varepsilon_{x y}, \tau_{x y x}=g^{2} \frac{E_{f}}{1+v_{f}} \frac{\partial \varepsilon_{x y}}{\partial x}, \tau_{x y y}=g^{2} \frac{E_{f}}{1+v_{f}} \frac{\partial \varepsilon_{x y}}{\partial y}
\end{aligned}
$$

where $\varepsilon_{x x}=-z \frac{\partial^{2} w}{\partial x^{2}}, \varepsilon_{y y}=-z \frac{\partial^{2} w}{\partial y^{2}}, \varepsilon_{x y}=-z \frac{\partial^{2} w}{\partial x \partial y}$. And $w$ is the deflection of the plate. $\mathrm{E}_{\mathrm{f}}$ and $v_{f}$ are the elastic modulus and Poisson's ratio of the film, respectively. The strain energy of the gradient elastic plate reads

$$
\begin{aligned}
U= & \frac{1}{2} \iiint \sigma_{x x} \varepsilon_{x x}+\sigma_{y y} \varepsilon_{y y}+2 \sigma_{x y} \varepsilon_{x y} d x d y d z \\
& +\frac{1}{2} \iiint \tau_{x x x} \varepsilon_{x x x}+2 \tau_{x y x} \varepsilon_{x y x}+\tau_{y y x} \varepsilon_{y y x} \\
& +\tau_{x x y} \varepsilon_{x x y}+2 \tau_{x y y} \varepsilon_{x y y}+\tau_{y y y} \varepsilon_{y y y} d x d y d z
\end{aligned}
$$

The variation of the work done by the external forces has the form (Papargyri-Beskou et al., 2010; Timoshenko and WoinowskyKrieger, 1959):

$$
\begin{aligned}
\delta W= & \iint_{A}(q-p) \delta w d x d y-\int_{s} M_{n} \frac{\partial \delta w}{\partial n} d s \\
& +\int_{s} V_{n} \delta w d s-\int_{s} M_{n n} \frac{\partial^{2} \delta w}{\partial n^{2}} d s
\end{aligned}
$$


where $\mathrm{q}$ is the force of the indenter on the film, and $\mathrm{p}$ is the reaction force of the substrate to the film. $M_{n}$ and $M_{n n}$ are the bending moment and the higher-order moment of the boundary, respectively, and $V_{n}$ is the total shear force. The variational formulation for the gradient elastic plate mounted on a compliant substrate can be established as follows:

$\delta U-\delta W=0$

Substitution of Eqs. (2), (3) and (4) into Eq. (5), the bending governing equation of the gradient elastic film mounted on a compliant substrate can be obtained

$D \nabla^{4} w(x, y)-g^{2} D \nabla^{6} w(x, y)=q(x, y)-p(x, y)$

where $D=\frac{E_{f} t_{f}^{3}}{12\left(1-v_{f}^{2}\right)}$ is the bending stiffness of the film. $\nabla^{4} w(x, y)$ and $\nabla^{6} w(x, y)$ are given by

$\nabla^{4} w=\frac{\partial^{4} w}{\partial x^{4}}+\frac{\partial^{4} w}{\partial y^{4}}+2 \frac{\partial^{4} w}{\partial x^{2} \partial y^{2}}$

$\nabla^{6} w=\frac{\partial^{6} w}{\partial x^{6}}+\frac{\partial^{6} w}{\partial y^{6}}+3 \frac{\partial^{6} w}{\partial x^{4} \partial y^{2}}+3 \frac{\partial^{6} w}{\partial x^{2} \partial y^{4}}$

For an axisymmetric gradient elastic film mounted on a compliant substrate, Eq. (6) can be expressed as

$D \nabla^{4} w(r)-g^{2} D \nabla^{6} w(r)=q(r)-p(r)$

where $\nabla^{4} w(r)$ and $\nabla^{6} w(r)$ are given by

$\nabla^{4} w=\frac{d^{4} w}{d r^{4}}+\frac{2}{r} \frac{d^{3} w}{d r^{3}}-\frac{1}{r^{2}} \frac{d^{2} w}{d r^{2}}+\frac{1}{r^{3}} \frac{d w}{d r}$

$\nabla^{6} w=\frac{1}{r} \frac{d}{d r}\left(r \frac{d}{d r} \nabla^{4} w\right)$

2.2. The relationship between the deflection and the indentation load

Based on the Hankel transformation, the relationship between the deflection and the indentation load of the stiff film/compliant substrate system can be derived by combining Eq. (8) and the solution for the semi-infinite elastic foundation under an axisymmetric load. The Hankel transformation of Eq. (8) can be expressed as

$D \xi^{4} \bar{w}(\xi)+g^{2} D \xi^{6} \bar{w}(\xi)=\bar{q}(\xi)-\bar{p}(\xi)$

where $\bar{w}(\xi), \bar{q}(\xi)$, and $\bar{p}(\xi)$ are the zero-order Hankel transformation of $w(\mathrm{r}), \mathrm{q}(\mathrm{r})$, and $\mathrm{p}(\mathrm{r})$, respectively. At the same time, the relationship between loads and surface displacements of a semiinfinite space under an arbitrary symmetric load is (Tan and Guo, 2016)

$w(r)=\frac{2\left(1-v_{s}^{2}\right)}{E_{s}} \int_{0}^{+\infty} \bar{p}(\xi) J_{0}(\xi r) d \xi$

where $E_{S}$ and $v_{s}$ are the elastic modulus and Poisson's ratio of the substrate. Using Eq. (10) in Eq. (11), we can get

$w(r)=\frac{2\left(1-v_{s}^{2}\right)}{E_{s}} \int_{0}^{+\infty}\left[\bar{q}(\xi)-D \xi^{4} \bar{w}(\xi)-g^{2} D \xi^{6} \bar{w}(\xi)\right] J_{0}(\xi r) d \xi$

In addition, according to the Hankel transformation, $w(\mathrm{r})$ can also be expressed as

$w(r)=\int_{0}^{+\infty} w(\xi) J_{0}(\xi r) \xi d \xi$

Comparing Eq. (12) and Eq. (13), we can easily get

$\bar{w}(\xi)=\frac{2\left(1-v_{s}^{2}\right)}{E_{s}} \frac{\bar{q}(\xi)}{\xi\left[1+l^{3} \xi^{3}+(g / l)^{2} l^{5} \xi^{5}\right]}$ where $l=\sqrt[3]{\frac{2 D\left(1-v_{s}^{2}\right)}{E_{s}}}$. Then, the Hankel inverse transformation is performed on Eq. (14), and $w(r)$ has the form

$w(r)=\frac{2\left(1-v_{s}^{2}\right)}{E_{s}} \int_{0}^{+\infty} \frac{\bar{q}(t / l)}{1+t^{3}+(g / l)^{2} t^{5}} J_{0}\left(\frac{t}{l} r\right) d t$

where $t=l \xi$. At this point, it is easy to know that the relationship between indentation load and displacement can be got by calculating $\bar{q}(t / l)$. Assuming that the pressure between the indenter and the film satisfies the Hertz distribution (according to the reference (Liu et al., 2019b), this assumption is reasonable), namely, q(r) can be expressed as

$q(r)=\frac{3 Q}{2 \pi a^{2}}\left[1-\left(\frac{r}{a}\right)^{2}\right]^{1 / 2}, r \leq a ; q(r)=0, r>a$

The Hankel transformation of $\mathrm{q}(\mathrm{r})$ can be written as

$\bar{q}(\xi)=\frac{3 Q}{2 \pi a^{2}} \int_{0}^{a}\left[1-\left(\frac{r}{a}\right)^{2}\right]^{1 / 2} J_{0}(\xi r) r d r$

Letting $\xi=t / l, \rho=r / l, \alpha=a / l$, then Eq. (17) becomes

$\bar{q}\left(\frac{t}{l}\right)=Q \frac{3}{2 \pi \alpha^{2}} \int_{0}^{\alpha}\left[1-\left(\frac{\rho}{\alpha}\right)^{2}\right]^{1 / 2} J_{0}(t \rho) \rho d \rho$

Using Eq. (18) in Eq. (15), we can get

$w(r)=\frac{Q}{D^{1 / 3}}\left[\frac{2\left(1-v_{s}^{2}\right)}{E_{s}}\right]^{2 / 3} \int_{0}^{+\infty} \frac{I_{1}(\alpha, t)}{1+t^{3}+\left(g_{/ l}\right)^{2} t^{5}} J_{0}\left(t \frac{r}{l}\right) d t$

where $I_{1}(\alpha, t)=\frac{3}{2 \pi \alpha^{2}} \int_{0}^{\alpha}\left[1-\left(\frac{\rho}{\alpha}\right)^{2}\right]^{1 / 2} J_{0}(t \rho) \rho d \rho$. For the convenience of writing, Eq. (19) can be written as

$w(r)=I_{2}\left(\alpha, \frac{r}{l}\right) \frac{Q}{D^{1 / 3}}\left[\frac{2\left(1-v_{s}^{2}\right)}{E_{s}}\right]^{2 / 3}$

where $I_{2}\left(\alpha, \frac{r}{l}\right)=\int_{0}^{+\infty} \frac{I_{1}(\alpha, t)}{1+t^{3}+(g / l) t^{2}} J_{0}\left(t \frac{r}{l}\right) d t$. Eq. (20) describes the relationship between total load and displacement at any point on the film. When $\mathrm{g}$ is 0 , Eq. (20) degenerates into the traditional indentation solution of the stiff film/compliant substrate system with a high modulus ratio. When $r$ is taken as 0 , the relationship between the indentation load and the depth can be got:

$w(0)=I_{2}(\alpha, 0) \frac{Q}{D^{1 / 3}}\left[\frac{2\left(1-v_{s}^{2}\right)}{E_{s}}\right]^{2 / 3}$

\subsection{The bending moment on the gradient elastic film}

In order to simplify the solution process of the bending moment on the gradient elastic film, we may consider the load due to the indenter as a point load (It is proved that this will not introduce obvious errors in Section 4). Namely, $\alpha$ approaches 0 infinitely. At this time, $I_{1}$ approaches $1 / 2 \pi$. In the above case, $w(\mathrm{r})$ can be written as

$w(r)=I_{2}\left(\frac{r}{l}\right) \frac{Q}{D^{1 / 3}}\left[\frac{2\left(1-v_{s}^{2}\right)}{E_{s}}\right]^{2 / 3}$

where $I_{2}\left(\frac{r}{l}\right)=\int_{0}^{+\infty} \frac{1}{1+t^{3}+(g / l)^{2} t^{5}} J_{0}\left(t \frac{r}{l}\right) d t$. Then the first derivative and the second derivative of $w(r)$ with respect to $\mathrm{r}$ can be expressed as

$\frac{d w(r)}{d r}=\frac{Q}{2 \pi D^{2 / 3}}\left[\frac{2\left(1-v_{s}^{2}\right)}{E_{s}}\right]^{1 / 3} I_{3}\left(\frac{r}{l}\right)$ 
$\frac{d^{2} w(r)}{d r^{2}}=\frac{Q}{2 \pi D} I_{4}\left(\frac{r}{l}\right)$

where $I_{3}\left(\frac{r}{l}\right)$ and $I_{4}\left(\frac{r}{l}\right)$ are explicitly given by

$$
\begin{aligned}
& I_{3}\left(\begin{array}{l}
r \\
l
\end{array}\right)=\int_{0}^{+\infty} \frac{t}{1+t^{3}+(g / l)^{2} t^{5}}\left[-J_{1}\left(t \frac{r}{l}\right)\right] d t \\
& I_{4}\left(\begin{array}{l}
r \\
l
\end{array}\right)=\int_{0}^{+\infty} \frac{t}{1+t^{3}+(g / l)^{2} t^{5}}\left[\frac{J_{1}\left(t_{l}^{r}\right)}{r / l}-t J_{0}\left(t \frac{r}{l}\right)\right] d t
\end{aligned}
$$

The radial bending moment and the circumferential bending moment on the film can be calculated by

$$
\begin{aligned}
& M_{r}(r)=-D_{\text {nor }}\left[\frac{d^{2} w(r)}{d r^{2}}+v_{f} \frac{1}{r} \frac{d w(r)}{d r}\right] ; \\
& M_{\varphi}(r)=-D_{\text {nor }}\left[\frac{1}{r} \frac{d w(r)}{d r}+v_{f} \frac{d^{2} w(r)}{d r^{2}}\right]
\end{aligned}
$$

It should be noted that the nominal bending stiffness, $D_{\text {nor }}$, is not the bending stiffness defined in the theory of plate, and its expression is given as follows:

Eq. (20) is expressed in the form of traditional solutions:

$w(r)=I_{2}^{g=0}\left(\alpha, \frac{r}{l}\right) \frac{Q}{D_{\text {nor }} 1 / 3}\left[\frac{2\left(1-v_{s}^{2}\right)}{E_{s}}\right]^{2 / 3}$

where $D_{\text {nor }}{ }^{1 / 3}$ and $I_{2}^{g=0}\left(\alpha, \frac{r}{l}\right)$ are explicitly given by

$D_{\text {nor }}^{1 / 3}=\left(I_{2}^{g=0}\left(\alpha, \frac{r}{l}\right) / I_{2}\left(\alpha, \frac{r}{l}\right)\right) D^{1 / 3}$

$I_{2}^{g=0}\left(\alpha, \frac{r}{l}\right)=\int_{0}^{+\infty} \frac{I_{1}(\alpha, t)}{1+t^{3}} J_{0}\left(t \frac{r}{l}\right) d t$

\section{The gradient effect on the indentation response of the stiff film/compliant substrate system}

In this section, the influences of the gradient effect on the indentation response of the stiff film/compliant substrate system were investigated from three aspects: the load-displacement relationship, the surface profiles and the bending moment distribution on the film. In addition, based on the above three aspects of analysis, the core characteristics of the indentation gradient effect on the stiff film/compliant substrate composite structure are summarized. What needs to be added is that the validity of the solution is shown in the appendix, and from the appendix, we can know the indentation depth range should be less than the film thickness. Therefore, the indentation depth range will not be discussed in this section.

Based on the dimensional analysis, we can know that the load $Q /\left(E_{s} t_{f}^{2}\right)$ is a function of $E_{f} / E_{s}, v_{f}, v_{s}, g / t_{f}, \alpha$ and $w_{0} / t_{f}$ from Eq. (21): $Q /\left(E_{s} t_{f}^{2}\right)=f\left(E_{f} / E_{s}, v_{f}, v_{s}, g / t_{f}, w_{0} / t_{f}, \alpha\right)$. Here, the relationships between the indentation load and displacement at different modulus ratios, $E_{f} / E_{s}$, and different characteristic lengths, $g / t_{f}$, are analyzed. Fig. 2 shows the relationship between the indentation load and the displacement at different modulus ratios and different characteristic lengths. All results were obtained at $\alpha=0.25$. The reason for this is that for the stiff film/compliant substrate system with a high modulus ratio, $\alpha$ is generally less than 0.5 (Liu et al., 2019a), and the load-displacement relationship is not sensitive to $\alpha$, which will be verified in Section 4. From Fig. 2 we can know the load increases linearly with the indentation depth. However, the slope of the line at different modulus ratios of the film to the substrate or $g / t_{f}$ is different from each other. Fig. 2(a) shows the results at different modulus ratios, from which we can know that the indentation load increases as the modulus ratio becomes large at the same depth. For example, when the dimensionless indentation depth is 0.5 , the dimensionless load increases by 4.36 times when the modulus ratio changes from $10^{2}$ to $10^{5}$, which is easy to understand. For example, we assume that the modulus of the substrate is a constant, then, the higher the modulus ratio the larger the bending stiffness of the film. Therefore, at the same depth, the higher the modulus ratio the larger the indentation load. Fig. 2(b) shows the results at different characteristic lengths of the solid. What should be emphasized that for common metal materials (e.g. $\mathrm{Cu}, \mathrm{Ni}, \mathrm{Fe}), \mathrm{g}$ is about a few microns no matter whether it is a case of the strain gradient elasticity or plasticity ((Aifantis, 1999; Fleck et al., 1994; Lam et al., 2003; Song et al., 2014; Stölken and Evans, 1998), and the gradient theory is effective when the size is greater than the submicron level $(>0.1 \mu \mathrm{m})$. So, the range of $\mathrm{g} / \mathrm{t}_{\mathrm{f}}$ is about 0 to 100 ( $\mathrm{g}$ is taken as $1 \sim 10 \mu \mathrm{m}$, and $t_{f}$ is greater than 0.1$)$. In our study, the range of $g / t_{f}$ is taken as $0 \sim 40$. It can be seen from Fig. 2(b) that when $g / t_{f}$ becomes large, the slope of the load-depth relationship steeps, which is similar to the case where the modulus ratio increases (see Fig. 2(a)). For example, the slopes of the line are $3.87,4.95,5.32,5.97$ and 7.51 when $g / t_{f}$ are 0,10 , $13.3,20$ and 40, respectively. The reason for this is that due to the presence of the characteristic length of material $(g \neq 0)$, the nominal elastic modulus of the film increases with the decrease of the film thickness. Based on the above analysis, we can draw an important conclusion that because of the gradient effect, the characteristics of the load-displacement relationship of the stiff film/compliant substrate system changes when the film thickness decreases, moreover, the trend of the change is the same as the effect of increasing the modulus ratio of the film to the substrate.

From Eq. (20) we can know that the displacement of the point on the film, $w(r) / t_{f}$, is a function of $E_{f} / E_{s}, v_{f}, v_{s}, g / t_{f}, \alpha$ and $Q /\left(E_{s} t_{f}^{2}\right): w(r) / t_{f}=f\left(E_{f} / E_{s}, v_{f}, v_{s}, g / t_{f}, \alpha, Q /\left(E_{s} t_{f}^{2}\right)\right)$. Here, the profiles of the film at different modulus ratios and different characteristic lengths under a constant load are analyzed. Fig. 3 shows the deflection profiles of stiff film surface when the indentation depth, $w_{0} / t_{\mathrm{f}}$, is 1 based on Eq. (20) with $\alpha=0.25$. This is because the surface morphology of the film is also not sensitive to $\alpha$, which will be verified in Section 4. Fig. 3(a) shows the results at different modulus ratios, from which we can know the greater the modulus ratio, the flatter the surface morphology of the film. The reason for this is that the higher the modulus ratio, the greater the bending stiffness of the film, and then the range of the sink-down of the surface is greater under the same displacement load. Fig. 3(b) shows the results at different characteristic lengths of the film. As shown in Fig. 3(b), as $g / t_{f}$ increases, the surface morphology of the film tends to be flat, which is similar to the change in the surface morphology of the film as the modulus ratio increases. The reason for this is that due to the gradient effect, the nominal modulus of the film becomes large when $\mathrm{g} / \mathrm{t}_{\mathrm{f}}$ increases, which leads to the similarity of the change trends of topography between the case where the modulus ratio increases and the case where $\mathrm{g} / \mathrm{t}_{\mathrm{f}}$ increases. The above analysis reminds us that when the film thickness is reduced, the gradient effect changes the characteristic of the surface morphology of the film, and the changing trend is similar to the change caused by the increase of the modulus ratio.

From Eq. (25) we can know the radial bending moment, $M_{r} /\left(E_{s} t_{f}^{2}\right)$, and the circumferential bending moment, $M_{\varphi} /\left(E_{s} t_{f}^{2}\right)$, are a function of $E_{f} / E_{s}, v_{f}, v_{s}, g / t_{f}, \alpha$ and $Q /\left(E_{s} t_{f}^{2}\right): M_{r} /\left(E_{s} t_{f}^{2}\right)=f\left(E_{f} / E_{s}, v_{f}, v_{s}, g / t_{f}, \alpha, Q /\left(E_{s} t_{f}^{2}\right)\right)$ and $M_{\varphi} /\left(E_{s} t_{f}^{2}\right)=f\left(E_{f} / E_{s}, v_{f}, v_{s}, g / t_{f}, \alpha, Q /\left(E_{s} t_{f}^{2}\right)\right)$. Here, the bending moment at different modulus ratios and different characteristic lengths under a constant load are analyzed. Fig. 4 shows the bending moment distribution on the film based on Eq. (25). Fig. 4(a) and (b) are for the radial bending moment at different modulus 

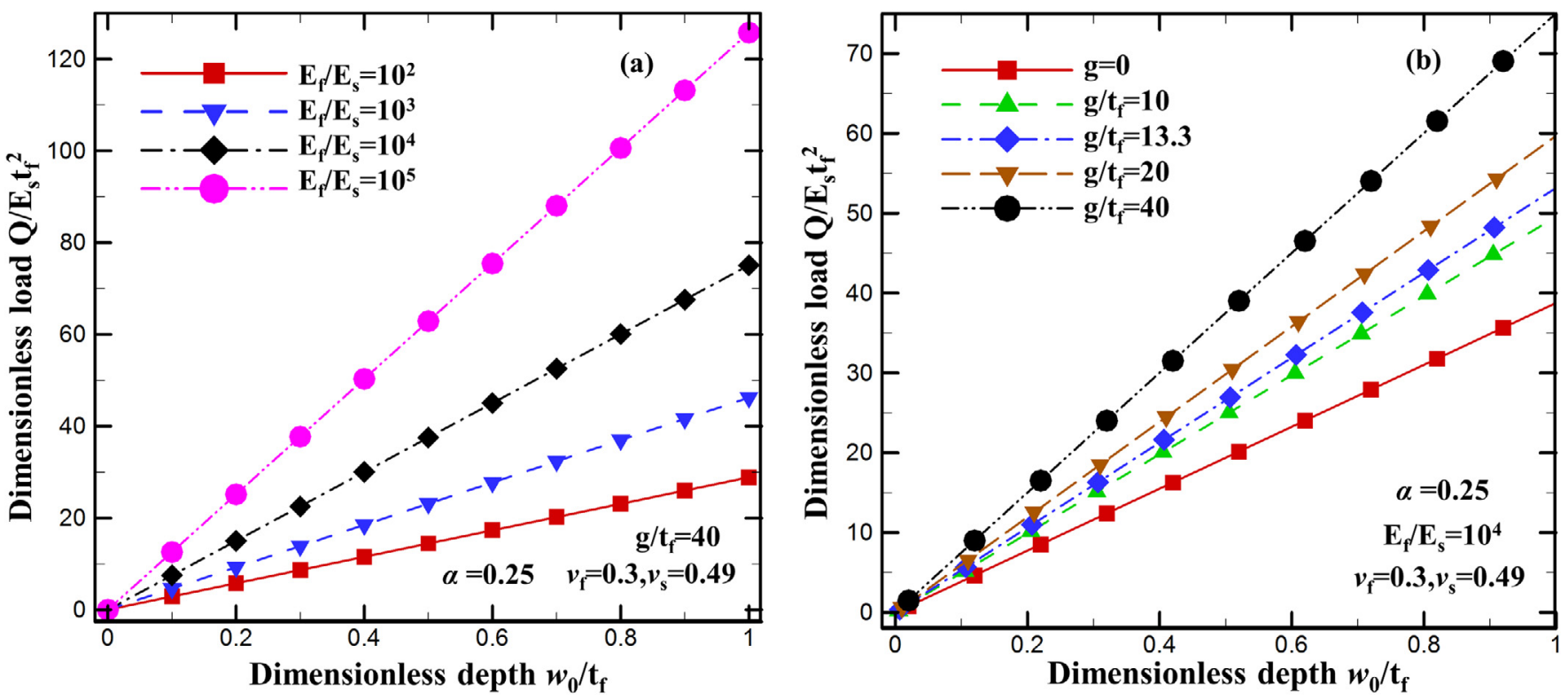

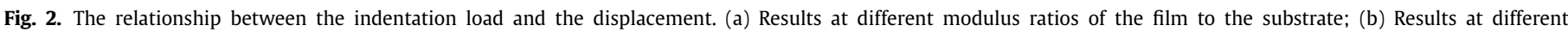
characteristic lengths of the film.
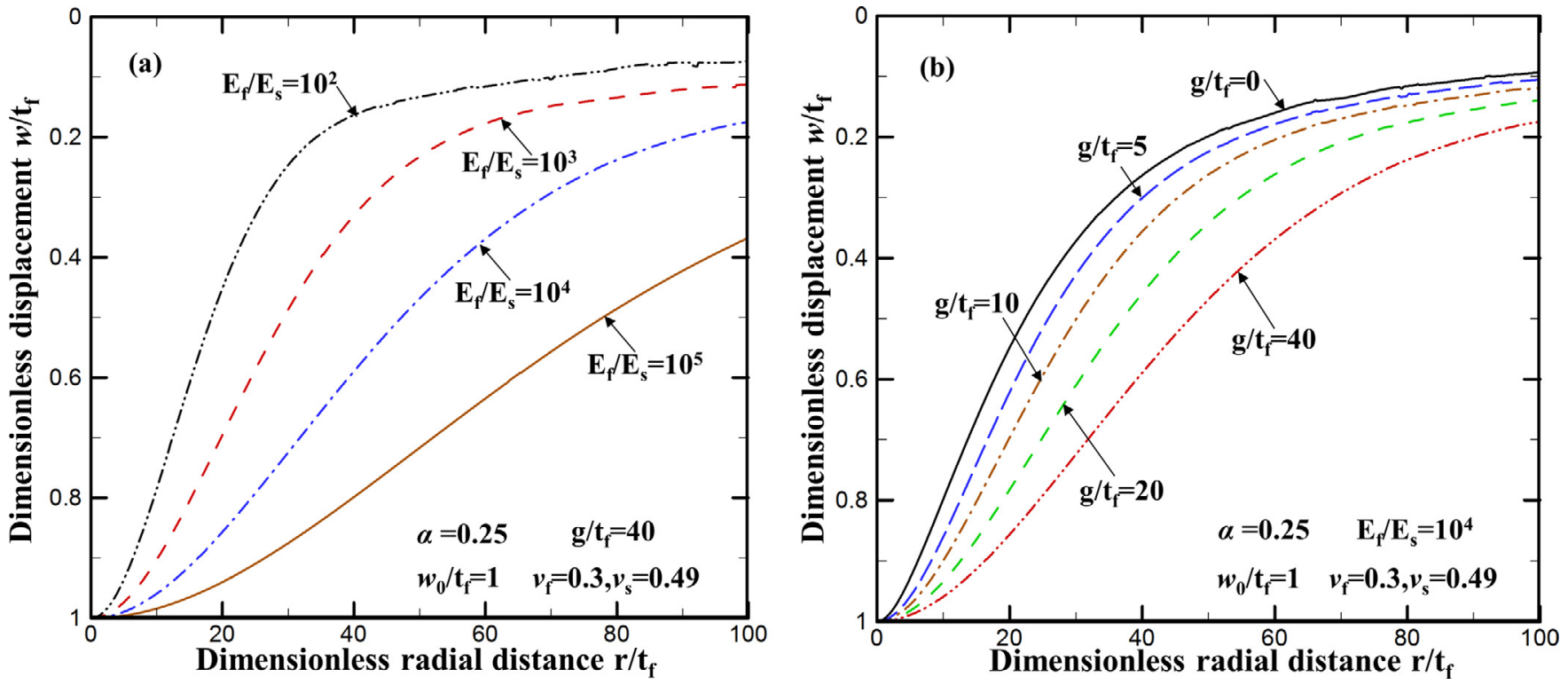



ratios and characteristics lengths, and Fig. 4(c) and (d) are for the circumferential bending moment at different modulus ratios and characteristics lengths. As shown in Fig. 4(a) and (b), along the radial direction of the film, the radial bending moment first decreases to zero and then increases to the maximum in the negative direction and finally decreases to zero. Taking the modulus ratio of the film to the substrate equals $10^{4}$ as an example, the radial bending moments are $7.80,-1.35$ and -0.03 when the radial distances are $0,60.50$ and 200, respectively. At different modulus ratios or $\mathrm{g} / \mathrm{t}_{\mathrm{f}}$, the maximum radial bending moment in the positive and negative directions are different, and the point on the film at which the maximum radial bending moment in the negative direction appears is also different. From Fig. 4(a) we can know when the modulus ratio is gradually increased, the maximum radial bending moment in the positive direction increases, and the maximum radial bending moment in the negative direction becomes large, but the point on the film at which the maximum radial bending moment in the negative direction appears is more and more delayed. For example, the maximum radial bending moments in positive directions are 2.80, 4.62, 7.80 and 14.08 and the maximum radial bending moments in negative directions are $-0.51,-0.83,-1.35$ and -2.22 when the modulus ratios of the film to the substrate are $10^{2}, 10^{3}, 10^{4}$ and $10^{5}$, respectively. And the abscissa of the points on the film at which the maximum radial bending moment in the negative direction appears are $22.84,37.97,60.84$ and 94.7995 when the modulus ratios of the film to the substrate are $10^{2}, 10^{3}, 10^{4}$ and $10^{5}$, respectively. What's more, the variation of the radial bending moment along the radial direction of the film at different modulus ratios is similar. In contrast, at different $\mathrm{g} / \mathrm{t}_{\mathrm{f}}$, the variation of radial bending moment 

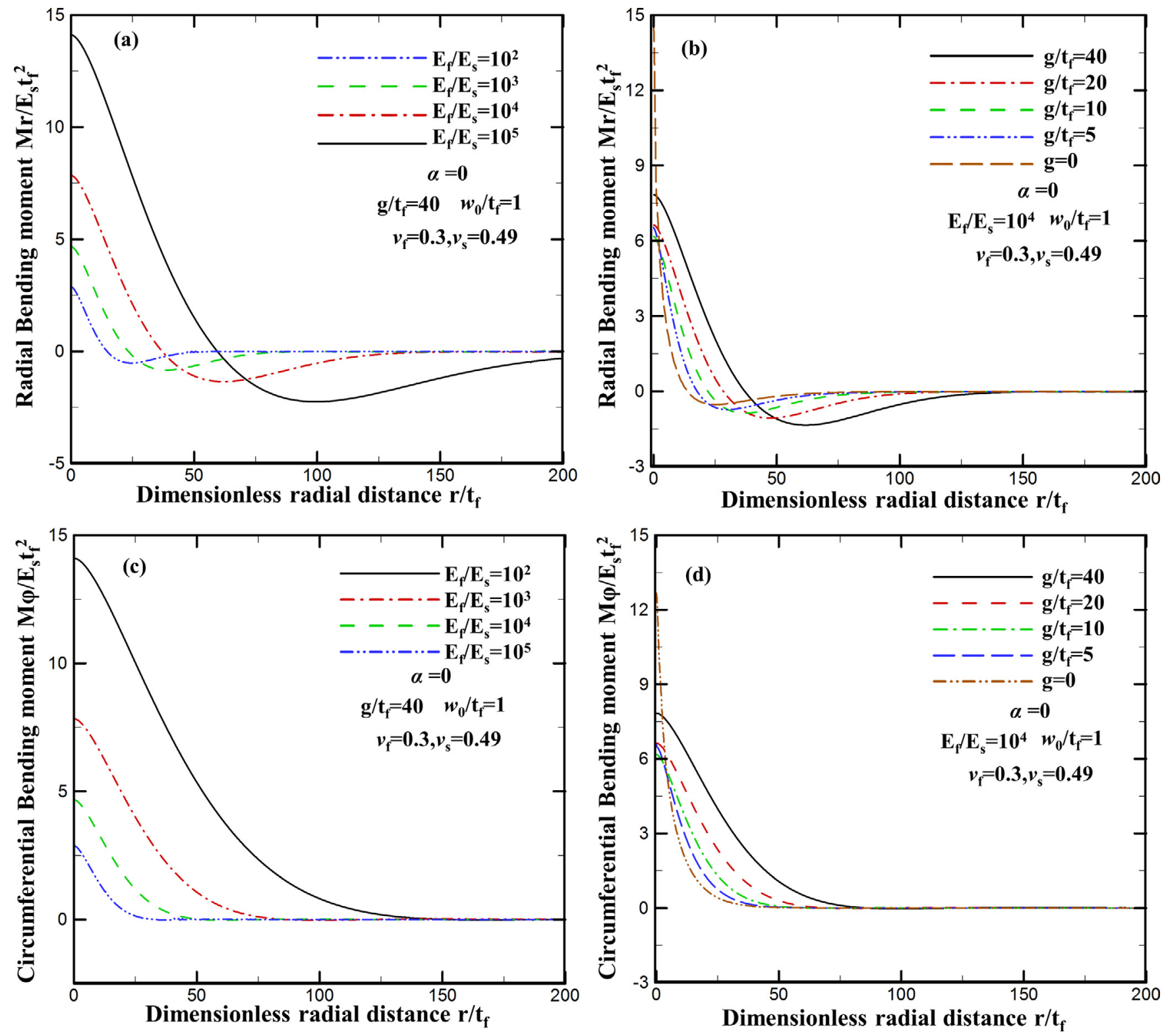

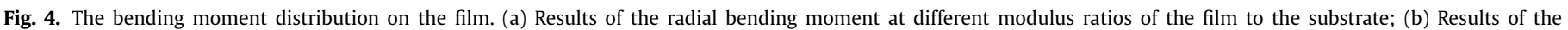

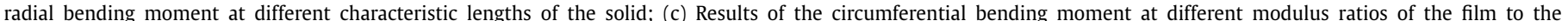
substrate; (d) Results of the circumferential bending moment at different characteristic lengths of the solid.

in the radial direction does not have such similarity, which is shown in Fig. 4(b). It can be seen from Fig. 4(b) that when $g / t_{f}$ increases, the maximum radial bending moment in the positive direction first decreases, and then increases, which is defferent from the case where the modulus ratio increases. For example, the maximum radial bending moments in positive directions are 13.78, 6.49, 6.17, 6.63 and 7.84 when $\mathrm{g} / \mathrm{tf}$ are $0,5,10,20$ and 40 , respectively. Moreover, the maximum bending moment in the negative direction increases with the increase of $\mathrm{g} / \mathrm{t}_{\mathrm{f}}$, and the point on the film at which the maximum radial bending moment in the negative direction appears is more and more delayed, which is the same as the case where the modulus ratio increases.

Unlike the radial bending moment, the circumferential bending moment monotonically decreases along the radial direction of the film, which is shown in Fig. 4(c). when the modulus ratio increases, the maximum circumferential bending moment in the positive di- rection becomes large. For example, the circumferential bending moment increases by 4.96 times when the modulus ratio changes from $10^{2}$ to $10^{5}$. And the point on the film at which the circumferential bending moment decreases to zero is more and more delayed as the modulus ratio becomes larger. Besides, the variation of the circumferential bending moment along the radial direction of the film at different modulus ratios is similar. In contrast, at different $\mathrm{g} / \mathrm{t}_{\mathrm{f}}$, the variation of circumferential bending moment along the radial direction does not have such similarity, which is shown in Fig. 4(d). It can be seen from Fig. 4(d) that when $g / t_{f}$ increases, the maximum circumferential bending moment in the positive direction first decreases, and then increases, which is different from the case where the modulus ratio increases. For example, the maximum circumferential bending moments in the positive direction are $12.21,6.47,6.16,6.62$, and 7.83 when $\mathrm{g} / \mathrm{tf}$ are 0 , $5,10,20$ and 40, respectively. Based on the above analysis, we find that due to the existence of the gradient effect, the characteristic 

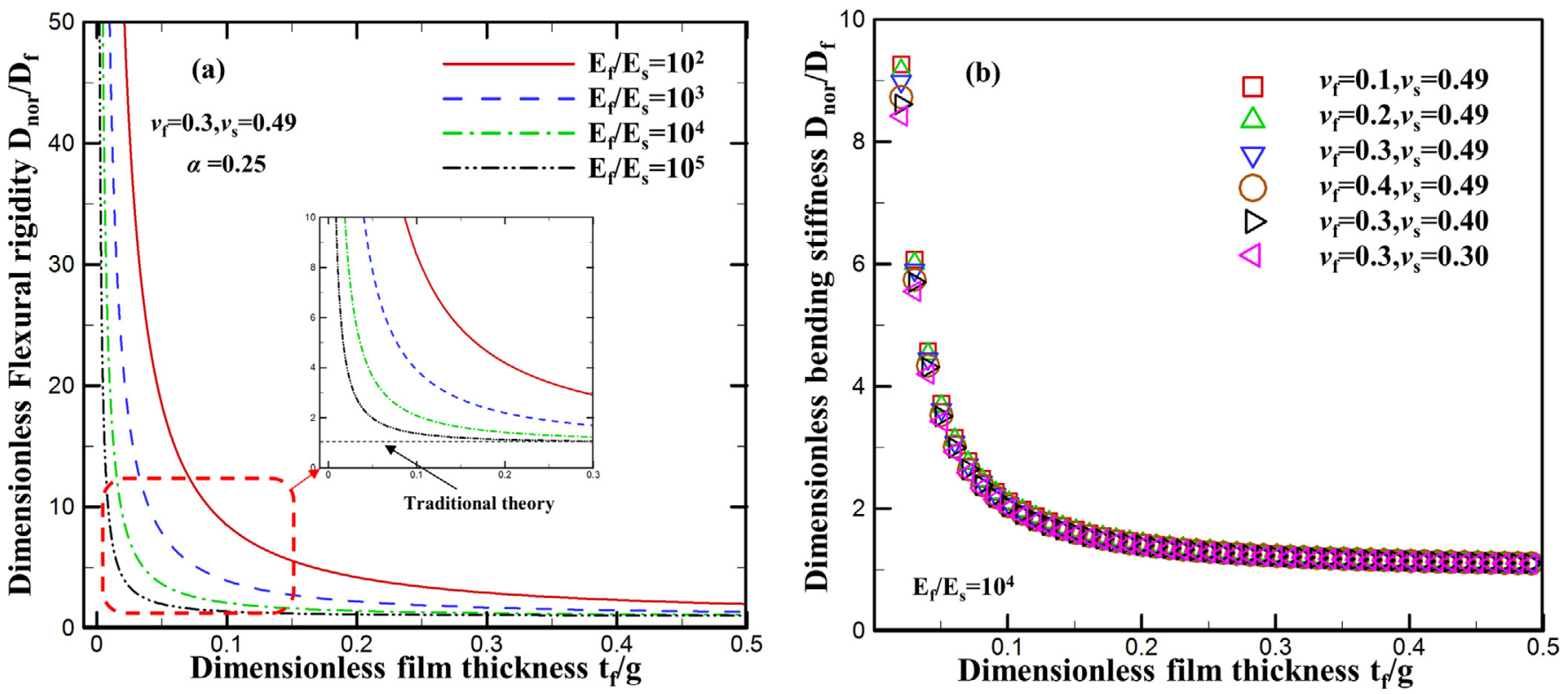

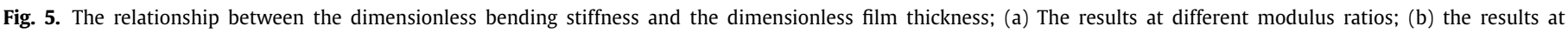
different Poisson's ratios.

of the bending moment distribution on the film changes when the film thickness decreases, and the changing trend is different from the case where the modulus ratio increases. The reason for this is that when the film thickness decreases, the nominal modulus of the film increases. However, the increase in nominal modulus and the reduction in film thickness are not coordinated, which results in the absence of similarities in the tendency of bending moments that change with the dimensionless radial distance at different $\mathrm{g} / \mathrm{t}_{\mathrm{f}}$.

Through the analysis of the above three aspects, it can be found that as the film thickness decreases, the indentation characteristics of the stiff film/compliant substrate composite structure change. This change caused by the decrease of the film thickness is similar to, but not identical to, the change caused by the increases of the modulus ratio of the film to the substrate. The reason for this is that the increase in the nominal modulus of the film and the decrease in the film thickness are not coordinated, thereby results in a nonlinear change in the bending stiffness of the film. In addition, it should be emphasized that due to the existence of size effects, the indentation behavior of the ultra-thin stiff film/compliant substrate composite structure cannot be described by the traditional theory, and the strain gradient theory should be used to predict the indentation behaviors.

\section{Analysis of factors influencing gradient effect}

Based on the analysis in Section 3, it can be known that when the film thickness is reduced, the nominal elastic modulus of the film is increased (in other words the dimensionless bending stiffness, $D_{n o r} / D$, is increased), thereby affecting the indentation response characteristics of the film/substrate composite structure. In other words, the dimensionless bending stiffness, $D_{\text {nor }} / D$, can represent the obvious degree of indentation gradient effect. In this section, the influences of the film thickness, modulus ratio, contact radius, and Poisson's ratio on the gradient effect are investigated. Based on the analysis of factors influencing the gradient effect, a dimensionless number, $\mathrm{g} / \mathrm{l}$, is proposed to evaluate the gradient effect.

Fig. 5 shows the variation of the dimensionless bending stiffness with respect to the dimensionless film thickness at different modulus ratios and Poisson's ratios. As can be seen from Fig. 5, the dimensionless bending stiffness is increased as the film thickness decreases, which implies the thinner the film thickness, the more obvious the gradient effect. However, the influences of the modulus ratio and Poisson's ratio on the gradient effect are different. From Fig. 5(a), we can know the variation of the dimensionless bending stiffness with respect to the film thickness at different modulus ratios is different. As shown in Fig. 5(a), when the modulus ratio increases, the dimensionless bending stiffness decreases at the same film thickness, which seems to indicate the inhibition of the modulus ratio on the gradient effect. For example, when the dimensionless indentation depth is 0.05 , the dimensionless bending stiffness is $18.61,7.93,3.68$ and 2.00 when the modulus ratio changes from $10^{2}$ to $10^{5}$, respectively. The above phenomenon shows that gradient effects are related not only to film thickness but also to the modulus ratio. Compared with the modulus ratio, the influence of Poisson's ratio on the gradient effect is small. It can be seen from Fig. 5(b) that the variation of the dimensionless bending stiffness with respect to the film thickness is basically unchanged at different Poisson's ratios.

The influence of the indentation depth and the shape of the indenter on the indentation gradient effect has been extensively studied (Chen et al., 2007; Saha et al., 2001; Zhang et al., 2007). Here, in order to investigate the influence of the indentation depth and the shape of the indenter, we use different contact radii to represent different indentation depths or indenter shapes, which is reasonable (it is well known that when indenter shapes do not change, the deeper the indentation depth the larger the contact radius; or when the indentation depth is not changed, the bigger the indenter radius the larger the contact radius). In addition, for the stiff film/compliant substrate system with a high modulus ratio $\left(E_{f} / E_{s}>10^{2}\right)$, the contact radius is often less than 0.5 (Liu et al., 2019a). Therefore, we will only discuss the case where the contact radius is less than 0.5. As can be seen from Fig. 6(a), the relationship between the bending stiffness and the film thickness at different contact radii almost coincides. Namely, the contact radius has little effect on the dimensionless bending stiffness. In addition, since the magnitude of the contact radius can represent the magnitude of the indentation depth (as you know, the greater the indentation depth, the larger the contact radius), it can be seen that the gradient effects are the same at different indentation depth, which 



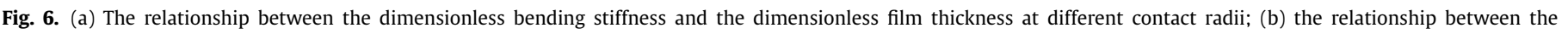
indentation load and depth at different contact radii; (c) the deflection profiles of the film at different contact radii.

is different from the stiff film/compliant substrate system with a low modulus ratio $\left(E_{f} / E_{s}<10\right)$. The reason for this is that the deformation mechanism that controls the two systems is different. The mechanism of the indentation behavior of the stiff film/compliant substrate system having a high modulus ratio is the bending of the film, while that of the stiff film/compliant substrate system with low modulus ratio is mainly Hertz contact. And Fig. 5(b) and (c) also reflect the insignificance of the influence of contact radius on the gradient effect. For example, at different contact radii, the loaddepth relationship or the deflection profiles of the film almost coincides with each other.

From the above analysis, we find that the gradient effect is not sensitive to the contact radius and Poisson's ratio, but is sensitive to the film thickness and the modulus ratio of the film to the substrate. Combining this finding with Eq. (21), a dimensionless number, $g / l$, is proposed to describe the indentation gradient effect of the stiff film/compliant substrate system with a high modulus ratio. As shown in Fig. 7, the values of the dimensionless bending stiffness under different modulus ratios, $\mathrm{t}_{\mathrm{f}} / \mathrm{g}$, and $\alpha$ are on the line which is the relationship between the dimensionless bending stiffness and the dimensionless number $\mathrm{g} / \mathrm{l}$ defined by us. Based on the fact that the gradient effect on the indentation response of the stiff film/compliant substrate composite structure can be described by dimensionless numbers $\mathrm{g} / \mathrm{l}$, we propose a method for measuring the characteristic length of materials in the laboratory. The steps of the method are described as follows: firstly, depositing the nano or micro film on a compliant substrate with known properties (e.g. PDMS); then, the indentation test is performed to obtain the load-displacement relationship; thirdly, the nominal bending stiffness, $D_{\text {nor }}$, of the film is obtained by fitting the loaddisplacement relationship based on Eq. (26); finally, calculating the dimensionless bending stiffness $D_{\text {nor }} / D$ and obtaining the value of the dimensionless number $\mathrm{g} / \mathrm{l}$ at this time in combination with Fig. 7. After obtaining the value of the dimensionless number, the characteristic length, $\mathrm{g}$, of the material can be obtained by a simple calculation. 


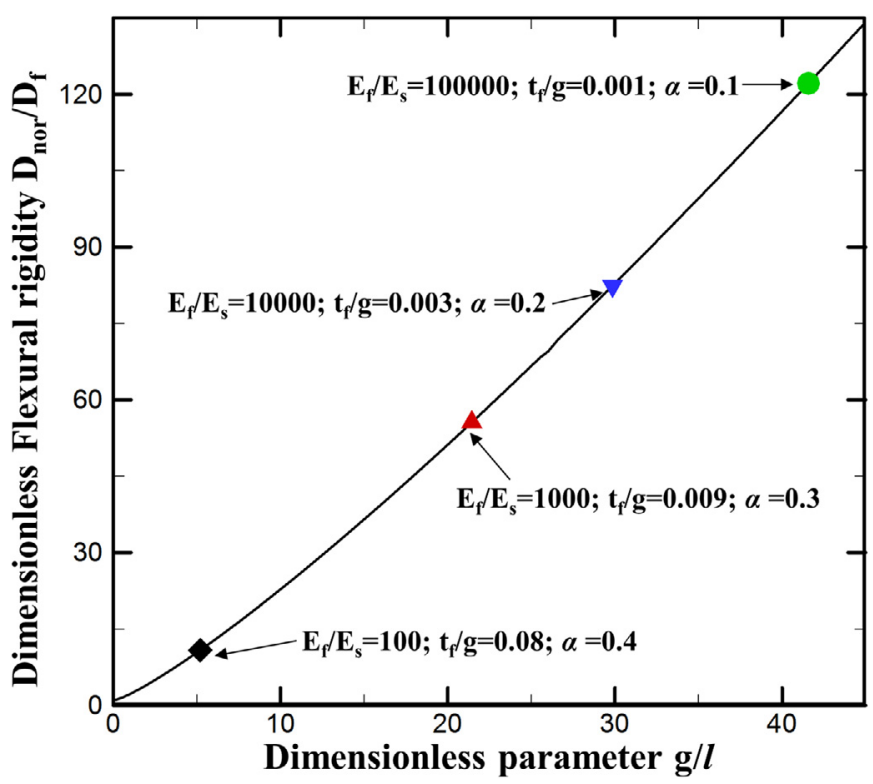

Fig. 7. The relationship between the dimensionless bending stiffness and the dimensionless number defined by us.

\section{Conclusion}

The gradient effect on the indentation response of the stiff film/compliant substrate composite structure is studied from a theoretical perspective. Based on a simple strain gradient theory and the theory of plate, a closed-form indentation solution of a gradient elastic stiff film mounted on a soft elastic substrate is obtained with the help of Hankel transformation under the assumption that the contact stress satisfies the Hertz distribution. The influence of the gradient effect on the indentation response is discussed from three aspects: the load-displacement relationship, the surface topography and the distribution of the bending moment on the film. The results show that due to the existence of the gradient effect, when the film thickness is reduced, the indentation characteristics of this composite structure change. The change in the indentation behavior caused by the reduction in the film thickness is similar to, but not identical to, the change resulting from an increase in the modulus ratio of the film to the substrate. And we further studied the factors that influence the gradient effect, which includes the modulus ratio, film thickness, contact radius, and Poisson's ratio. It is found that the gradient effect is almost unaffected by the contact radius and Poisson's ratio. However, interestingly, the gradient effect due to the reduction in film thickness is affected not only by the thickness of the film but also by the modulus ratio of the film to the substrate. In addition, when the modulus ratio is increased, the gradient effect is weakened. Considering the couple influences of the film thickness and modulus ratio on the gradient effect, a dimensionless number, $\mathrm{g} / \mathrm{l}$, is proposed to describe the apparent extent of the gradient effect, and the results show that this dimensionless number can be very effective. Based on the fact that the gradient effect of the system can be described by the dimensionless number $\mathrm{g} / \mathrm{l}$, a new method for measuring the characteristic length of materials in the laboratory is proposed, which is more convenient and simpler than other methods for testing the characteristic length of materials. Our research provides a theoretical basis for an in-depth understanding of the gradient effects on the indentation response of the stiff film/compliant substrate system and for the measurement of the characteristic length of materials in the laboratory.

\section{Acknowledgements}

This work is supported by the National Natural Science Foundation of China under grants no. 11890681, 11672301, 11521202, 11432014 and 11572329.

\section{Appendix}

\section{(1) A detailed explanation of $\tau$}

$\tau_{i j k}$ is the couple stress. The first subscript, $\mathrm{i}$, designates the normal to the surface across which the component force acts; the second subscript, $\mathrm{j}$, designates the direction of the arm of the force; the third subscripts, $\mathrm{k}$, designates the direction of the force (Mindlin, 1964). For example, $\tau_{123}$ is shown in Fig. A1:

(2) The analysis of the validity range of the closed-form solution with $g=0$

Inspired by Xia et al. (Xia et al., 2007), we analyzed the validity range of our solutions with $g=0$ based on the theory of plate. Based on our analysis, we can know that the performance of the gradient effect is to improve the nominal elastic constant of materials. Therefore, the validity range of solution with $g=0$ can provide a valid reference for the solution with $g \neq 0$ (Note: the scope of traditional solutions is used as a reference, not as a guideline). In addition, it can be known that when $g=0$, our solution degenerates into the traditional solution. At this time, we can take

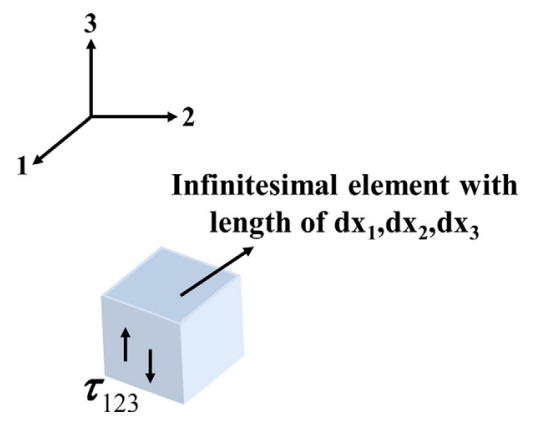

Fig. A1. Component, $\tau_{123}$, of double stress.

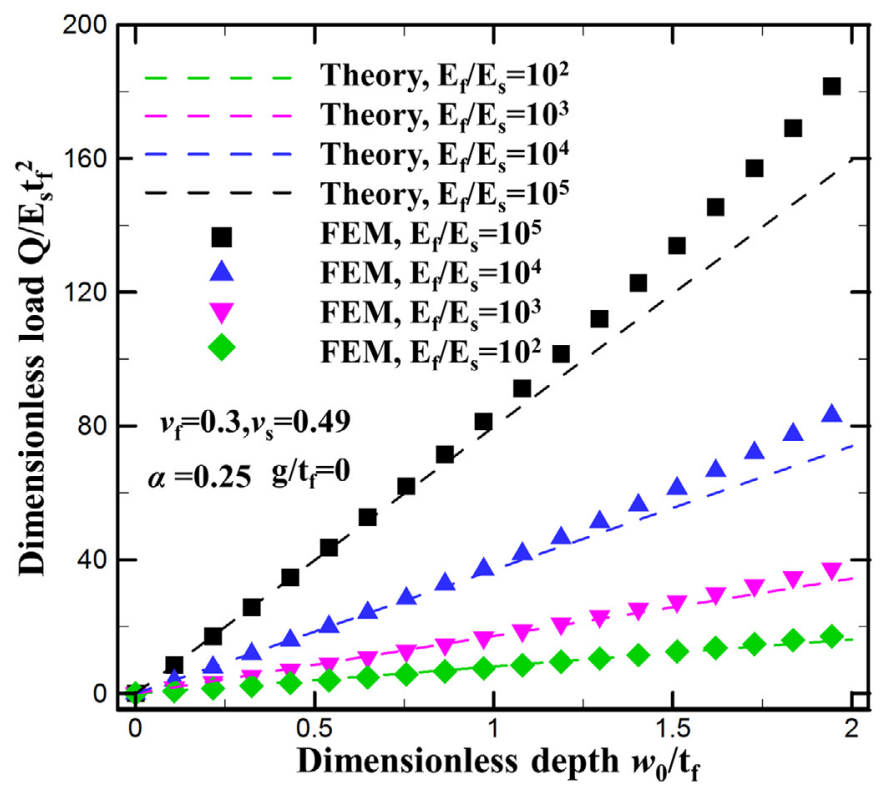

Fig. A2. The relationship between the indentation depth and the load. The lines are for the theory with $g=0$, and the symbols are for the FEM. 
the FEM simulation results as a benchmark to evaluate the validity range of our solution with $g=0$. Fig. A2 shows the comparison between the theory and the FEM results. From the figure, we can know for the stiff film/compliant substrate system with a high modulus ratio $\left(E_{\mathrm{f}} / \mathrm{E}_{\mathrm{s}}>10^{2}\right)$, when the indentation depth, $\mathrm{w}_{0} / \mathrm{t}_{\mathrm{f}}$, is less than 1, the error between the theory and the FEM is less than $5 \%$. Based on the above analysis, it can be seen that our solution with $g=0$ is effective when the indentation depth is less than the film thickness. Based on the above analysis, the applicable indentation range of our model should be less than the film thickness, but the specific application range needs to be further verified by means of experiments or simulations.

In addition, what we should emphasize is that because we consider the indentation response of the stiff film/compliant substrate system as the bending of an infinite gradient elastic plate mounted on a semi-infinite elastic foundation, the indentation size effect of the system is caused by the decrease of film size, not by the decrease of the indentation depth. The detailed analysis for this can be found in Section 4 of this paper or in previous studies (Kahrobaiyan et al., 2011; Papargyri-Beskou and Beskos, 2008; Wang et al., 2011).

\section{References}

Aifantis, E.C., 1999. Strain Gradient Interpretation of Size effects, Fracture Scaling. Springer, pp. 299-314.

Altan, B., Aifantis, E., 1997. On some aspects in the special theory of gradient elasticity. J Mech Behav Mater 8, 231-282.

Balint, D., Deshpande, V., Needleman, A., Van der Giessen, E., 2006. Discrete dislocation plasticity analysis of the wedge indentation of films. J Mech Phys Solids 54, 2281-2303.

Begley, M., Hutchinson, J.W., 1998. The mechanics of size-dependent indentation. J Mech Phys Solids 46, 1029.

Bucaille, J.L., Felder, E., Hochstetter, G., 2002. Identification of the viscoplastic behavior of a polycarbonate based on experiments and numerical modeling of the nano-indentation test. J Mater Sci 37, 3999-4011.

Burnett, P.J., Rickerby, D., 1987. The mechanical properties of wear-resistant coatings: I: modelling of hardness behaviour. Thin Solid Films 148, 41-50.

Cao, G., Liu, Y., Niu, T., 2018. Indentation response of two-dimensional materials mounted on different substrates. International Journal of Mechanical Sciences 137, 96-104.

Chen, S., Liu, L., Wang, T., 2007. Small scale, grain size and substrate effects in nano-indentation experiment of film-substrate systems. Int J Solids Struct 44, 4492-4504.

Chen, Z., Lu, H., 2012. Constructing sacrificial bonds and hidden lengths for ductile graphene/polyurethane elastomers with improved strength and toughness. J Mater Chem 22, 12479.

Doerner, M.F., Nix, W.D., 2011. A method for interpreting the data from depth-sensing indentation instruments. J Mater Res 1, 601-609.

Fang, M., Zhang, Z., Li, J., Zhang, H., Lu, H., Yang, Y., 2010. Constructing hierarchically structured interphases for strong and tough epoxy nanocomposites by amine-rich graphene surfaces. J Mater Chem 20, 9635.

Fleck, N., Hutchinson, J.W., 1997. In: Hutchinson, J W, Wu, T Y (Eds.). In: Strain Gradient Plasticity, 33. Advances in Applied Mechanics, p. 295 361 ed..

Fleck, N., Muller, G., Ashby, M., Hutchinson, J., 1994. Strain gradient plasticity: theory and experiment. Acta Metallurgica et Materialia 42, 475-487.

Fu, K., Chang, L., Ye, L., Yin, Y., 2016. Indentation stress-based models to predict fracture properties of brittle thin film on a ductile substrate. Surface and Coatings Technology 296, 46-57.

Gao, H., Cheng-Hsin, C., Jin, L., 1992. Elastic contact versus indentation modeling of multi-layered materials. Int J Solids Struct 29, 2471-2492.

Gao, H., Huang, Y., Nix, W., Hutchinson, J., 1999. Mechanism-based strain gradient plasticity-I. theory. J Mech Phys Solids 47, 1239-1263.

Gao, Y.F., Xu, H.T., Oliver, W.C., Pharr, G.M., 2008. Effective elastic modulus of film-on-substrate systems under normal and tangential contact. J Mech Phys Solids 56, 402-416.

Ho, D.H., Sun, Q., Kim, S.Y., Han, J.T., Kim, d.H., Cho, J.H., 2016. Stretchable and multimodal all graphene electronic skin. Advanced Materials 28, 2601.

Hou, C., Wang, H., Zhang, Q., Li, Y., Zhu, M., 2014. Highly conductive, flexible, and compressible all-graphene passive electronic skin for sensing human touch. Advanced Materials 26, 5018-5024.
Jönsson, B., Hogmark, S., 1984. Hardness measurements of thin films. Thin Solid Films 114, 257-269.

Kahrobaiyan, M., Asghari, M., Rahaeifard, M., Ahmadian, M., 2011. A nonlinear strain gradient beam formulation. Int J Eng Sci 49, 1256-1267.

King, R.B., 1987. Elastic analysis of some punch problems for a layered medium. Int J Solids Struct 23, 1657-1664.

Lam, D.C., Yang, F., Chong, A., Wang, J., Tong, P., 2003. Experiments and theory in strain gradient elasticity. J Mech Phys Solids 51, 1477-1508.

Lesage, J., Chicot, D., Pertuz, A., Jouan, P.-.Y., Horny, N., Soom, A., 2005. A model for hardness determination of thin coatings from standard micro-indentation tests. Surface and Coatings Technology 200, 886-889.

Liu, Y., Wei, Y., Chen, P., 2019a. Characterization of mechanical properties of two-dimensional materials mounted on soft substrate. International Journal of Mechanical Sciences 151, 214-221.

Liu, Y., Wei, Y., Chen, P., 2019b. Indentation response of soft viscoelastic matter with hard skin. Soft Matter 15, 5760-5769.

Mindlin, R.D., 1964. Micro-structure in linear elasticity. Arch Ration Mech Anal 16, 51-78.

Niu, T., Cao, G., Xiong, C., 2018. Indentation behavior of the stiffest membrane mounted on a very compliant substrate: graphene on pdms. Int J Solids Struct 132-133, 1-8.

Papargyri-Beskou, S., Beskos, D., 2008. Static, stability and dynamic analysis of gradient elastic flexural kirchhoff plates. Archive of Applied Mechanics 78, 625-635.

Papargyri-Beskou, S., Giannakopoulos, A., Beskos, D., 2010. Variational analysis of gradient elastic flexural plates under static loading. Int J Solids Struct 47, 2755-2766.

Ramsey, P.M., Chandler, H.W., Page, T.F., 1991. Modelling the contact response of coated systems. Surf Coat Technol 49, 504-509.

Saha, R., Nix, W.D., 2002. Effects of the substrate on the determination of thin film mechanical properties by nanoindentation. Acta Mater 50, 23-38.

Saha, R., Xue, Z., Huang, Y., Nix, W.D., 2001. Indentation of a soft metal film on a hard substrate: strain gradient hardening effects. J Mech Phys Solids 49, 1997-2014.

Song, J., Liu, J., Ma, H., Liang, L., Wei, Y., 2014. Determinations of both length scale and surface elastic parameters for fcc metals. Comptes Rendus Mecanique 342, 315-325.

Stölken, J., Evans, A., 1998. A microbend test method for measuring the plasticity length scale. Acta Mater 46, 5109-5115.

Tan, Z., Guo, J., 2016. The unified solution of infinite plate on elastic foundation. Chinese Journal of Applied Mechanics.

Thobor-Keck, A., Lapostolle, F., Dehlinger, A., Pilloud, D., Pierson, J., Coddet, C., 2005 Influence of silicon addition on the oxidation resistance of crn coatings. Surface and Coatings Technology 200, 264-268.

Timoshenko, S.P., Woinowsky-Krieger, S., 1959. Theory of Plates and Shells. McGraw-hill.

Tuck, J.R., Korsunsky, A.M., Bull, S.J., Davidson, R.I., 2001. On the application of the work-of-indentation approach to depth-sensing indentation experiments in coated systems. Surface and Coatings Technology 137, 217-224.

Vanimisetti, S.K., Narasimhan, R., 2006. A numerical analysis of spherical indentation response of thin hard films on soft substrates. Int J Solids Struct 43 6180-6193.

Wang, B., Zhou, S., Zhao, J., Chen, X., 2011. A size-dependent kirchhoff micro-plate model based on strain gradient elasticity theory. European Journal of Mechanics-A/Solids 30, 517-524.

Wang, Q.H., Kalantar-Zadeh, K., Kis, A., Coleman, J.N., Strano, M.S. 2012. Electronics and optoelectronics of two-dimensional transition metal dichalcogenides. Nat Nanotechnol 7, 699.

Xia, S., Gao, Y., Bower, A.F., Lev, L.C., Cheng, Y.-T., 2007. Delamination mechanism maps for a strong elastic coating on an elastic-plastic substrate subjected to contact loading. Int J Solids Struct 44, 3685-3699.

Xu, H., Pharr, G.M., 2006. An improved relation for the effective elastic compliance of a film/substrate system during indentation by a flat cylindrical punch. Scr Mater 55, 315-318.

Xu, Y., Balint, D., Dini, D., 2019. A new hardness formula incorporating the effect of source density on indentation response: a discrete dislocation plasticity analysis. Surface and Coatings Technology 374, 763-773.

Yin, Z., Sun, S., Salim, T., Wu, S., Huang, X., He, Q., Lam, Y.M., Zhang, H., 2010. Organic photovoltaic devices using highly flexible reduced graphene oxide films as transparent electrodes. ACS Nano 4, 5263-5268.

Zhang, F., Saha, R., Huang, Y., Nix, W., Hwang, K., Qu, S., Li, M., 2007. Indentation of a hard film on a soft substrate: strain gradient hardening effects. International Journal of Plasticity 23, 25-43.

Zheludkevich, M., Serra, R., Montemor, M., Yasakau, K., Salvado, I.M., Ferreira, M. 2005. Nanostructured sol-gel coatings doped with cerium nitrate as pre-treatments for AA2024-T3: corrosion protection performance. Electrochim. Acta 51, 208-217. 